\begin{tabular}{|c|c|}
\hline Title & $\begin{array}{l}\text { Influence of suspension viscosity and colloidal particles on permeability of membrane used in membrane } \\
\text { bioreactor(MBR) }\end{array}$ \\
\hline Author(s) & Itonaga, Takanori; Kimura, Katsuki; W atanabe, Y oshimasa \\
\hline Citation & 衛生工学シンポジウム論文集, 11, 127-130 \\
\hline Issue Date & $2003-10-31$ \\
\hline Doc URL & http://hdl.handle.net/2115/7062 \\
\hline Type & bulletin (article) \\
\hline Note & 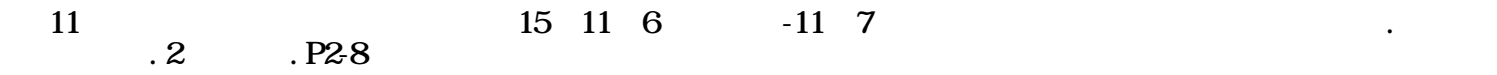 \\
\hline File Information & 11-2-8_p127-130.pdf \\
\hline
\end{tabular}

Instructions for use 


\title{
2-8 Influence of suspension viscosity and colloidal particles on permeability of membrane used in membrane bioreactor (MBR)
}

\author{
Takanori Itonaga*, Katsuki Kimura*, and Yoshimasa Watanabe* \\ * Department of Urban and Environmental Engineering, Hokkaido University, North-13, West-8, Kita-ku, Sapporo \\ 060-8628, Japan. E-mail: itonaga@eng.hokudai.ac.jp
}

\section{INTRODUCTION}

Application of submenged membrane bioreactors (MBRs) in which biological treatment and solid-liquid separation are simultaneously carried out in a single reaction chamber to wastewater treatment has received much attention recently. This is partly because high quality of treated water and smaller footprint can be expected by using a MBR, compared with other existing biological wastewater treatment processes such as activated sludge process. Main obstacle for wider application of MBRs is, however, deterioration of membrane permeability (membrane fouling) with operation time. Analysis of the particle size distribution of organic substances in raw municipal wastewater has shown that a large part of the organic matter in raw municipal wastewater (63-70\%, as measured by the TOC tests) is associated with particles that are larger than $0.1 \mu \mathrm{m}$, which can be easily removed by coagulation and sedimentation. Coagulation and sedimentation as the pre-treatment for MBRs dealing with municipal wastewater were examined by the authors and was found to considerably suppress membrane fouling. Also, by installing coagulation and sedimentation, quality of treated water from the MBRs was obviously improved.

In this study, pilot scale experiments were carried out to compare membrane fouling occurring in a MBR without pre-treatment (hereafter called conventional MBR (CMBR)) with fouling occurring in a MBR with pre-coagulation/sedimentation (hereafter called hybrid MBR (HMBR)). Especially, the influence of suspension viscosity and dissolved organic matter (DOM) on membrane fouling was investigated. A series of laboratory scale dead-end filtration experiments was also carried out to investigate which fraction (i.e., suspended solids (SS), colloidal matter and soluble matter) contained in biomass suspension would be the most influential in deterioration of membrane permeability.

\section{MATERIALS AND METHODS \\ Pilot scale MBR}

Fig.1 shows a schematic diagram of the pilot plant installed at an existing municipal wastewater treatment plant (Soseigawa treatment plant, Sapporo, Japan). This treatment plant receives wastewater collected by a combined sewer system. Effluent from the primary clarifier was used as the raw water in this study. Two long-term experiments (Runs 1 and 2) were conducted in this study. In Run 1, effect of installation of coagulation/sedimentation as the pre-treatment of MBR was examined. In addition, the optimum range of MLSS concentration was sought in the range between $3 \mathrm{~g} / \mathrm{L}$ and $25 \mathrm{~g} / \mathrm{L}$. In Run 2, influence of DOM on membrane permeability was investigated. Jet mixed separator (JMS) with inclined tube settlers was used for pre-coagulation/sedimentation. Flow rate and HRT in the JMS were set at $50 \mathrm{~m}^{3} /$ day and 1.5 hours, respectively. Iron based coagulant, poly-silicato iron (PSI), was used as coagulant. Dosage of PSI was fixed at $10 \mathrm{mg}-\mathrm{Fe} / \mathrm{L}$. Table 1 summarizes the operating conditions for each run. In both runs, two identical MBRs were operated with different feed water in parallel. Primary clarifier effluent was directly fed to CMBR while HMBR was fed with pre-coagulation/sedimentation. Hollow fiber MF membrane modules (Mitsubishi Rayon, Japan) were submerged in the membrane chambers and used for the experiments. Material of the membrane and effective membrane area of each module were

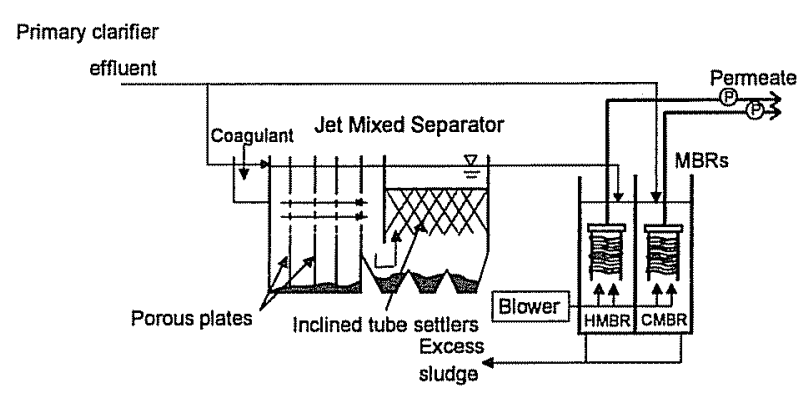

Fig. 1 Flow diagram of the pilot plant. 
polyethylene and $3 \mathrm{~m}^{2}$, respectively. The aeration devices were installed right below the membrane modules for removing cake layer and oxygen supply. Effective volume of each MBR was $0.18 \mathrm{~m}^{3}$. When deterioration in membrane permeability became significant, the membrane module was taken out from the membrane chamber and physically washed by spraying pressurized water.

Laboratory scale dead-end membrane filtration experiment A series of dead-end membrane filtration experiments was conducted to investigate which fraction in biomass suspension would be the most influential in deterioration of membrane permeability. Biomass suspensions collected from the MBRs during Run 2 were used in these experiments. Flat plate membranes were used for the dead-end tests. Nominal pore size and effective area of the used membrane were $0.1 \mu \mathrm{m}$ and $26.9 \mathrm{~cm}^{2}$, respectively. Operating pressure was maintained at $40 \mathrm{kPa}$. During the dead-end tests, the permeate flux was periodically monitored. By using the data obtained in the dead-end tests, membrane fouling caused by the suspensions was evaluated.

\section{RESULTS AND DISCUSSION}

Effect of installation of coagulation/sedimentation as pre-treatment for MBRs.

Run 1 was carried out to examine the effect of installation of coagulation/sedimentation as the pre-treatment for MBRs. Fig. 2 shows the changes in total membrane filtration resistance $\left(R_{t}\right)$ observed in Run 1. Permeate flux was varied during Run 1 and the variation of it is also shown in Fig.2. Fig.3 shows the changes in MLSS concentrations. In CMBR, rapid increase in $R_{f}$ was observed from the initiation of the operation. On 19 days of the operation, the membrane module was taken out from CMBR and physical membrane washing (i.e., spraying pressurized water) was carried out. However, no significant restoration in membrane permeability was recognized and consequently chemical membrane cleaning was needed. After chemical cleaning, rapid increase in $R_{t}$ of CMBR was observed again and the second membrane cleaning had to be carried out. After day 130, permeate flux of CMBR is increased to the same flux as that of HMBR. As a result, rapid increase in $R_{t}$ was frequently observed in CMBR. When the membrane module was taken out from CMBR for physical cleaning, formation of thick and sticky cake layer (i.e., deposition of biomass suspension) on the membrane surface was recognized. In contrast with the results with $\mathrm{CMBR}$, relatively high membrane permeability was maintained with $\mathrm{HMBR}$. Much less frequency of membrane cleaning is required in the operation of HMBR. Recorded value of $R_{t}$ just after physical
Table 1 Operating conditions of the MBRs.

\begin{tabular}{llcc}
\hline & & Run 1 & Run 2 \\
\hline HRT in MBR & $(\mathrm{hr})$ & $3.6-6.0$ & 4.5 \\
MLSS conc. & $(\mathrm{g} / \mathrm{L})$ & $3-25$ & 10 \\
Permeate flux & $\left(\mathrm{m}^{3} / \mathrm{m}^{2} /\right.$ day $)$ & $0.3-0.5$ & 0.4 \\
Pore size & $(\mu \mathrm{m})$ & 0.4 & 0.2 \\
Operation & & Suction for $12 \mathrm{~min}$. \\
cycle & & Stop for $3 \mathrm{~min}$. \\
\hline
\end{tabular}

1. Physical membrane cleaning

隹 Chemical membrane cleaning

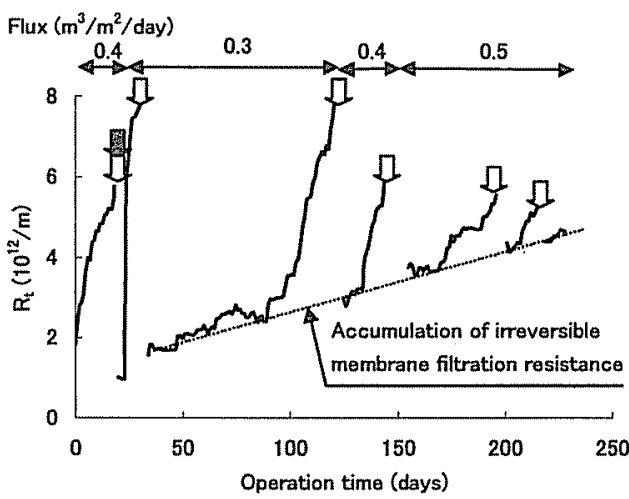

(a) CMBR

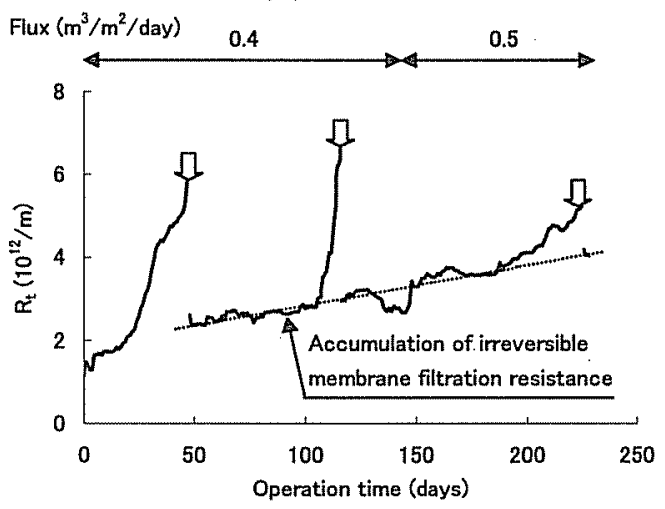

(b) HMBR

Fig.2 Changes in the total membrane filtration resistance $\left(R_{t}\right)$.

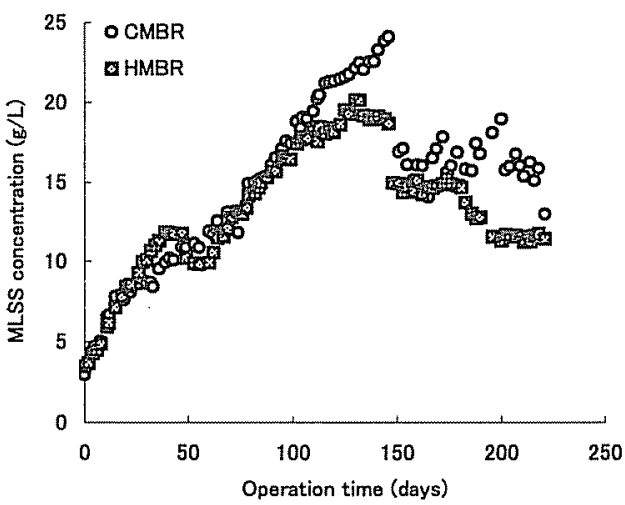

Fig.3 Changes in MLSS concentrations 
cleaning should represent the magnitude of irreversible fouling since physical cleaning was supposed to cancel reversible fouling. Based on this assumption, changes in irreversible fouling in Run 1 are also plotted in Fig.2 (dotted lines) by connecting $R_{t} s$ observed just after physical cleaning. As clearly shown in Fig.2, accumulation of irreversible membrane filtration resistance increased with operation time. Accumulation of irreversible resistance observed in HMBR was $40 \%$ less rapid than that in CMBR. From these results, it can be considered that pre-coagulation/sedimentation would improve the performance of MBR in terms of membrane permeability by controlling both formation of sticky cake layer on the membrane surface and irreversible fouling.

\section{Influence of suspension viscosity on membrane fouling in CMBR}

Relationship between MLSS concentration and suspension viscosity observed in Run 1 is shown in Fig.4. As clearly shown in Fig.4, suspension viscosity of CMBR was greater than that of HMBR especially in high MLSS concentration ranges. By referring to Fig.2 (a) and Fig.3, it can be seen that membrane permeability of CMBR was significantly deteriorated after 100 days of the operation when MLSS concentration was maintained above $15 \mathrm{~g} / \mathrm{L}$. Such a high MLSS concentration caused high suspension viscosity in CMBR. On the other hand, suspension viscosity observed in HMBR was maintained at a low level even when MLSS concentration was high (i.e., above $15 \mathrm{~g} / \mathrm{L}$ ). This difference in suspension viscosity in both MBRs probably explains the difference in evolution of $R_{i}$ (Fig.2). Namely, more rapid increase of $R_{t}$ in CMBR than HMBR was due to the high suspension viscosity in CMBR. Thus, in order to efficiently operate a MBR, suspension viscosity in the membrane chamber should be maintained at a low level. This can be achieved easily in the case of HMBR, implying the advantage of this process. Careful inspection of Fig.4 reveals that an inflection point where gradient of suspension viscosity of CMBR obviously changes exists around $10 \mathrm{~g}$-MLSS/L. When CMBR operation was conducted with MLSS concentrations well below the inflection

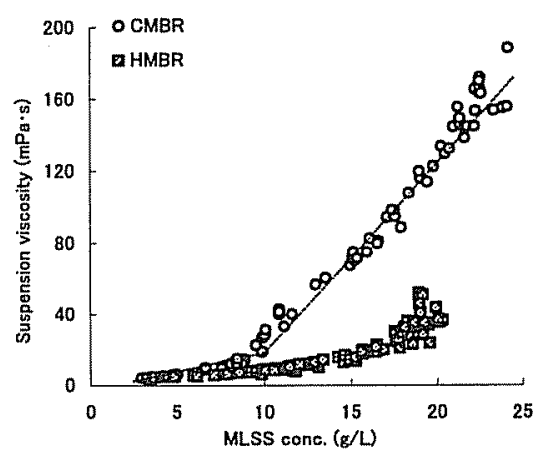

Fig.4 Relationship between MLSS concentration and suspension viscosity in the membrane chamber observed in Run 1.

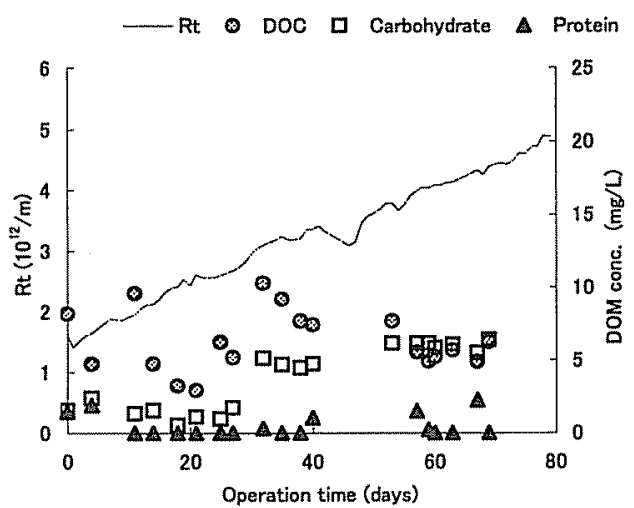
(a) CMBR

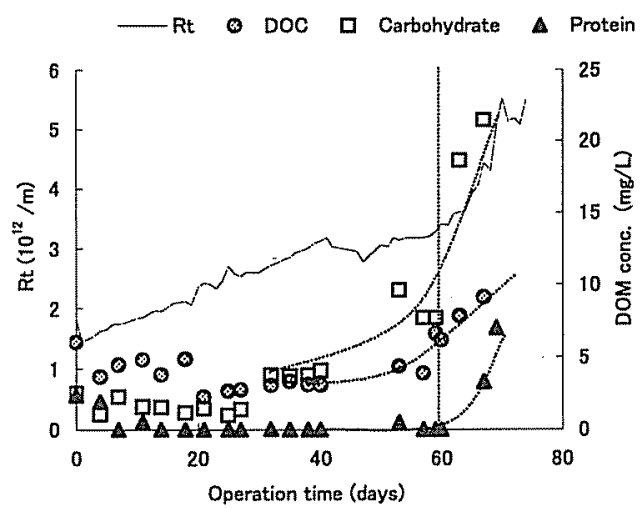

(b) HMBR

Fig. 5 Changes in the total-membrane fitration resistance $(R t)$ and DOM concentration in the membrane chamber.

point, formation of thick and sticky cake layer on the membrane surface was not observed. On the contrary, when MLSS concentration in CMBR was far beyond the inflection point (e.g., 15 g-MLSS/L), increase in suspension viscosity was considerable and immediate formation of the cake layer occurred. Based on these arguments, the optimum MLSS concentration for an efficient operation of MBRs was suggested to be around $10 \mathrm{~g} / \mathrm{L}$ in this study. When a MBR is used as the HMBR, higher MLSS concentration would be applicable.

\section{Influence of DOM/EPS on membrane permeability of MBRs}

Run 2 was carried out to investigate the influence of DOM on membrane permeability. Both CMBR and HMBR were examined in Run 2 as well. In Run 2, MLSS concentration in both MBRs was fixed at $10 \mathrm{~g} / \mathrm{L}$ based on the results described in the previous section. Fig.5 shows the changes in $R_{f}$ and DOM concentrations in the membrane chambers 
Table 2 Fouling resistances determined for each sample.

\begin{tabular}{|c|c|c|}
\hline Samples & CMBR & HMBR \\
\hline Biomass suspension (SS+colloid+soluble) & 2.8 & 6.0 \\
\hline After centrifugation ( $3000 \mathrm{rpm}, 5 \mathrm{~min}$ ) (colloid+soluble) & 2.3 & 5.3 \\
\hline After filtration $(0.5 \mu \mathrm{m})$ (soluble) & 1.8 & 1.5 \\
\hline
\end{tabular}

*unit=10 $12 / \mathrm{m}$

observed in Run 2. In Run 2, evolution of $R_{t}$ in both MBRs were suppressed compared with Run 1 probably because MLSS concentrations were kept at the appropriate value. Consequently, there was no need to carry out membrane cleaning throughout Run 2. Until 60 days of the operation, almost linear increase in $R_{f}$ was observed in both MBRs. The effect of pre-treatment for HMBR was confirmed again in Run 2. Namely, increase rate of $R_{i}$ observed in HMBR was $30 \%$ less rapid than that in CMBR until day 60 .

Until 60 days of the operation, DOC concentration in the membrane chamber fluctuated from 5 to $10 \mathrm{mg} / \mathrm{L}$ in CMBR while that in HMBR was stable around $5 \mathrm{mg} / \mathrm{L}$. Concentrations of dissolved carbohydrate in both membrane chambers were similar while dissolved protein was hardly detected in both membrane chambers. Higher DOC concentration in CMBR probably corresponded to more rapid evolution of $R_{t}$ in CMBR. Significant differences are not seen between CMBR and HMBR in terms of carbohydrate and protein, as shown in Fig.5. This implies that DOM that cannot be accounted for by carbohydrate and protein would be concerned with membrane fouling in MBRs.

\section{Role of suspended solids, colloidal matter and soluble matter in membrane fouling of MBRs}

A series of laboratory scale dead-end filtration experiments was carried out to examine the contributions of different fractions (i.e., SS, colloidal matter and soluble matter) contained in suspension to the total membrane fouling. Suspensions tested in the dead-end filtration were obtained from the membrane chambers of CMBR and HMBR at 68 days of the operation in Run 2 when the rapid membrane fouling occurred in HMBR. Calculation was based on the steady state (30 min of filtration) and results are summarized in Table 2 . From Table 2 , it can be seen that resistances caused by the suspension from HMBR were about two times higher than those determined for CMBR, except for the case after filtration. Comparing the resistances determined after filtration, it is found that soluble matter in HMBR suspension were less likely to cause fouling than that in CMBR. Comparing the three resistances shown in Table 2, degrees of resistances due to SS, colloidal matter and soluble matter are separately evaluated. Difference between the first row in Table 2 (Biomass suspension) and the second row (After centrifugation) represents the resistance due to SS. In a similar manner, the resistance due to colloidal matter can be represented. Values in the third row (After filtration) directly represent the resistance due to soluble matter. By carrying out this analysis, it was found that colloidal matter had the greatest fouling potential in the suspension collected from HMBR. The rapid increase in $R_{f}$ seen in Fig.5 (b) would be explained by these colloidal matter in the suspension.

\section{CONCLUSIONS}

In this study, pilot scale experiments were carried out to examine membrane fouling occurring in MBRs with or without pre-coagulation/sedimentation. Based on these experiments, the optimum MLSS concentration for efficient MBR operation and the influence of DOM on membrane fouling were investigated. A series of laboratory scale dead-end filtration experiments was also carried out to examine the contributions of particles with different sizes to the total membrane filtration resistance. The experimental results obtained in this study can be summarized as follows:

1. Pre-coagulation/sedimentation process improved the performance of a MBR in terms of membrane permeability by the control of both irreversible fouling and suspension viscosity in the membrane chamber.

2. The optimum MLSS concentration for efficient operation of a MBR was suggested to be around $10 \mathrm{~g} / \mathrm{L}$ beyond which substantial increase in suspension viscosity would be expected.

3. DOM that could not be accounted for by carbohydrate and protein seemed to be important in interpreting membrane fouling in MBRs.

4. Colloidal fraction in biomass suspension played an important role in membrane fouling. 